\title{
Mobile Phone Usability Questionnaire (MPUQ) and Automated Usability Evaluation
}

\author{
Young Sam Ryu \\ Ingram School of Engineering, \\ Texas State University-San Marcos, \\ 601 University Drive, San Marcos, TX 78666, USA \\ yryu@txstate.edu
}

\begin{abstract}
The mobile phone has become one of the most popular products amongst today's consumers. The Mobile Phone Usability Questionnaire (MPUQ) was developed to provide an effective subjective usability measurement tool, tailored specifically to the mobile phone. Progress is being made in the HCI research community towards automating some aspects of the usability evaluation process. Given that this effort is gaining traction, a tool for measurement of subjective usability, such as MPUQ, may serve as a complement to automated evaluation methods by providing user-centered values and emotional aspects of the product. Furthermore, experimental comparison of MPUQ assessments and automated usability analysis may enable researchers to determine whether automated usability tools generate metrics that correlate with user impressions of usability.
\end{abstract}

Keywords: Usability, mobile user interface, subjective measurement, questionnaire, automating usability.

\section{Development of MPUQ}

The mobile phone has become one of the most popular consumer products of today because it is suffused with personal meanings and individual experiences. It is carried from home to work and leisure activities, and it not only provides communication whenever needed, but can also act as a primary tool for life management [1-3]. Mobile phones have also been recognized as important indicators of consumers' tastes for buying other groups of products [4]. The mobile phone's design encompasses two major components (i.e., hardware and software), and aesthetic appeal and image may play an important aspect in evaluation of its usability. For these reasons, the mobile phone was selected as a worthwhile target-product for the development of a new usability questionnaire. The Mobile Phone Usability Questionnaire (MPUQ) was developed through two different phases. The goal and approach of each phase are described in Table 1.

The definition of usability in ISO 9241-11 was used to conceptualize the target construct, and the initial questionnaire items pool was comprised of material derived from various existing questionnaires, comprehensive usability studies, and other sources related to mobile devices. Through redundancy and relevancy analyses completed by representative mobile user groups, a total of 124 items (119 applicable to mobile phones 
and 115 applicable to PDA/Handheld PCs) were retained from an original 512 items in the initial pool. To increase reliability and validity of this draft questionnaire, follow-up studies employing psychometric theory and scaling procedures were performed. To evaluate the items, the draft questionnaire was administered to a representative sample involving approximately 300 participants. The findings revealed a six-factor structure including (1) Ease of learning and use, (2) Assistance with operation and problem solving, (3) Emotional aspect and multimedia capabilities, (4) Commands and minimal memory load, (5) Efficiency and control, and (6) Typical tasks for mobile phones. The 72 items with the greatest discriminative power relating to these factors were chosen to include in the Mobile Phone Usability Questionnaire (MPUQ), which evaluates mobile phones for the purpose of making decisions among competing variations in the end-user market, alternatives of prototypes during the development process, or evolving versions during an iterative design process.

Table 1. Development procedure of MPUQ

\begin{tabular}{|c|c|c|}
\hline Phase & Goal & Approach \\
\hline I & $\begin{array}{l}\text { Generate and judge } \\
\text { measurement items } \\
\text { for the usability } \\
\text { questionnaire for } \\
\text { electronic mobile } \\
\text { products }\end{array}$ & $\begin{array}{l}\text { Consider construct definition and content domain to develop } \\
\text { the questionnaire for the evaluation of electronic mobile } \\
\text { products based on an extensive literature review: } \\
\text { - Generate potential questionnaire items based on } \\
\text { essential usability attributes and dimensions for mobile } \\
\text { phone } \\
\text { - Judge items by consulting a group of experts and users } \\
\text { focusing on the content and face validity of the items }\end{array}$ \\
\hline II & $\begin{array}{l}\text { Design and } \\
\text { conduct studies to } \\
\text { develop and refine } \\
\text { the questionnaire }\end{array}$ & $\begin{array}{l}\text { Administer the questionnaire to collect data in order to refine } \\
\text { the items by } \\
\text { - Conducting item analysis via factor analysis } \\
\text { - Testing reliability using alpha coefficient } \\
\text { - Testing construct validity using known-group validity }\end{array}$ \\
\hline
\end{tabular}

\section{Automated Usability Evaluation and MPUQ}

Subjective usability measurements focus on an individual's personal experience with a product or system. According to Ivory and Hearst [5], automation of usability evaluation does not capture important qualitative and subjective information. However, it is not yet known whether subjective impressions of usability are in fact correlated with metrics that automated usability approaches can capture. By conducting subjective usability evaluation using a questionnaire of the same interface as has been modeled with an automated usability prediction tool such as CogTool [6], we can perhaps determine whether it may be the case that a metric such as time taken to complete tasks can be correlated with subjective impressions of usability.

One of the single greatest advantages of using questionnaires in usability research is that questionnaires can quickly and economically provide evaluators with feedback from the users' point of view [7-9]. Since user-centered and participatory design is one 
of the most important aspects in the usability engineering process [10], questionnaires, applied with or without any other more ambitious method, can be a valuable tool, assuming that the respondents are validated as representative of the whole user population.

There are many usability aspects or dimensions for which no established objective measurements exist, and those may only be measured by subjective assessment. New usability concepts suggested for the evaluation of consumer electronic products such as attractiveness [11], emotional usability [12], sensuality [13], pleasure and displeasure in product use [14] seem to be quantified effectively only by subjective assessment and those usability concepts are proving to be important these days. The MPUQ incorporated those dimensions; most of them are under the group of (3) Emotional aspect and multimedia capabilities. While other factor group items can be covered by other usability evaluation methods, the emotional aspects cannot presently be captured by any other practical approach than subjective measurement.

\section{References}

1. Vnnen-Vainio-Mattila, K., Ruuska, S.: Designing Mobile Phones and Communicators for Consumers' Needs at Nokia. In: Bergman, E. (ed.) Information Appliances and Beyond: Interaction Design for Consumer Products, pp. 169-204. Morgan-Kaufmann, San Francisco (2000)

2. Sacher, H., Loudon, G.: Uncovering the new wireless interaction paradigm. ACM Interactions Magazine 9(1), 17-23 (2002)

3. Ketola, P.: Integrating Usability with Concurrent Engineering in Mobile Phone Development. Tampereen yliopisto (2002)

4. PrintOnDemand. Popularity of Mobile Devices Growing (2003), http://www . printondemand. com/MT/archives/002021.html (cited February 5, 2003)

5. Ivory, M.Y., Hearst, M.A.: The state of the art in automating usability evaluation of user interfaces. ACM Comput. Surv. 33(4), 470-516 (2001)

6. John, B.E., et al.: Predictive human performance modeling made easy. In: The Proceedings of SIGCHI Conference on Human Factors in Computing Systems, CHI 2004, ACM, New York (2004)

7. Kirakowski, J.: Questionnaires in Usability Engineering: A List of Frequently Asked Questions [HTML] (2003) (cited November 26, 2003)

8. Annett, J.: Target Paper. Subjective rating scales: science or art? Ergonomics 45(14), 966987 (2002)

9. Baber, C.: Subjective evaluation of usability. Ergonomics 45(14), 1021-1025 (2002)

10. Keinonen, T.: One-dimensional usability - Influence of usability on consumers' product preference, University of Art and Design Helsinki, UIAH A21 (1998)

11. Caplan, S.H.: Making Usability a Kodak Product Differentiator. In: Wiklund, M. (ed.) Usability in Practice: How Companies Develop User-Friendly Products, pp. 21-58. Academic Press, Boston (1994)

12. Logan, R.J.: Behavioral and emotional usability; Thomson Consumer Electronics. In: Wiklund, M. (ed.) Usability in practice: How companies develop user friendly products, pp. 59-82. Academic press, Boston (1994)

13. Hofmeester, G.H., Kemp, J.A.M., Blankendaal, A.C.M.: Sensuality in product design: a structured approach. In: CHI 1996 Conference (1996)

14. Jordan, P.W.: Human factors for pleasure in product use. Applied Ergonomics 29(1), 25 33 (1998) 